\title{
Infrequent Intraprocedural Premature Ventricular Complexes: Implications for Ablation Outcome
}

\author{
KAZIM BASER, M.D., HATICE DUYGU BAS, M.D., MIKI YOKOKAWA, M.D., \\ RAKESH LATCHAMSETTY, M.D., FRED MORADY, M.D., and FRANK BOGUN, M.D.
}

From the Division of Cardiovascular Medicine, University of Michigan, Ann Arbor, Michigan, USA

\begin{abstract}
Premature Ventricular Complexes. Background: Frequent premature ventricular complexes (PVCs) can be eliminated with an ablation procedure. Ablation success rates have been reported to be in the $80 \%$ range. Reasons for failure of ablation have not been described in detail. The purpose of this study was to determine whether the paucity of PVCs at the beginning of the ablation procedure affects the outcome.

Methods: Catheter ablation was attempted in a consecutive series of 194 patients (age: $50 \pm 14$ years, 91 male, ejection fraction: $56.4 \pm \mathbf{8 . 4 \%}$ ) with frequent idiopathic PVCs. Based on receiver operator characteristics (ROC) analysis, patients were divided into 2 groups: Patients with frequent PVCs ( $\geq 32$ PVCs within the first 30 minutes of the procedure: $n=135$ [70\%]); and patients with infrequent PVCs ( $<32$ PVCs within the first 30 minutes of the procedure: $n=59[30 \%]$ ). Procedural outcomes were compared at 3 months postablation. A successful ablation was defined as a $\geq 80 \%$ reduction in the PVC burden compared to baseline.

Results: A successful procedure was performed in 148 patients $(\mathbf{7 6 \%})$ resulting in a decrease in the PVC burden from $19.1 \pm 13.6 \%$ to $0.38 \pm 0.98 \%(P<0.0001)$. Patients with frequent intraprocedural PVCs had a higher success rate than patients with infrequent intraprocedural PVCs $(85 \%$ vs. 56\%, $P=0.0001)$. Administration of sedation was no different in the 2 groups. The paucity of PVCs was independent of the site of origin in predicting procedural failure $(\mathrm{OR}: 6.9,95 \% \mathrm{CI}: 3.0-16.2 \mathrm{P}=0.0001)$.

Conclusion: Paucity of PVCs at the beginning of an ablation procedure is associated with a lower ablation success rate independent of the site of origin. (J Cardiovasc Electrophysiol, Vol. 25, pp. 1088-1092, October 2014)
\end{abstract}

infrequent PVCs, outcome, premature ventricular complexes

The reasons for failure of ablation of frequent premature ventricular complexes (PVCs) have not been detailed. Inability to reach the site of origin (SOO) may be a cause for failure, particularly in epicardial or intramural arrhythmias. ${ }^{1,2}$ Another cause for failure is the inability to identify the SOO because of imprecise mapping. In the absence of frequent PVCs, pacemapping is often used to identify the SOO. Pace-mapping is less precise than activation mapping. ${ }^{3}$ The purpose of this study was to assess whether the PVC prevalence at the onset of the procedure impacts procedural outcome.

\section{Methods}

\section{Patient Characteristics}

The subjects of this study consisted of a consecutive series of 194 patients, (103 women [53\%], mean age: $49.9 \pm$ 14.3 years, mean ejection fraction $[\mathrm{EF}]: 56.4 \pm 8.4 \%$ ) that were referred for catheter ablation (Table 1). Symptoms were

Dr. Bogun has received a grant from the Leducq Foundation.

No disclosures.

Address for correspondence: Frank Bogun, M.D., Cardiovascular Center, Division of Cardiovascular Medicine, University of Michigan, SPC 5853, 1500 E. Medical Center Drive, Ann Arbor, MI 48109-5853. Fax: 734-9367026; E-mail: fbogun@med.umich.edu

Manuscript received 18 March 2014; Revised manuscript received 23 April 2014; Accepted for publication 13 May 2014.

doi: $10.1111 /$ jce. 12454 absent in 35 patients, and 45 patients had PVC induced cardiomyopathy $(\mathrm{EF}<50 \%)$. The presence of coronary artery disease, hypertensive heart disease, valvular heart disease, or hypertrophic cardiomyopathy was ruled out by echocardiography, stress testing, coronary angiography, and/or cardiac magnetic resonance imaging. Patients had failed $0.8 \pm 0.4$ antiarrhythmic medications including $\beta$-blocker and calcium channel blocker therapy. All antiarrhythmic medications including $\beta$-blockers and calcium channel blockers were discontinued at least 5 half-lives prior to the ablation procedure. The mean number of PVC morphologies was $1.9 \pm 2.2$. There was no difference in the number of PVC morphologies between infrequent and frequent $\mathrm{PVC}$ groups $(\mathrm{P}=0.9)$. Upon presentation in the electrophysiology laboratory, the frequency of the predominant PVC was determined during the initial 30 minutes after the patient was connected to the ECG recording system. Patients were divided into 2 groups depending on the prevalence of PVCs. If the number of PVC within the first 30 minutes was $<32$ PVCs, the intraprocedural PVC burden was defined as infrequent (see below for the basis of this cut-off value).

\section{Holter Recordings}

All patients had a 12-lead 24-hour Holter monitor prior to the ablation procedure. The Holter was repeated 3 months postablation and success was defined as a reduction of the initial PVC burden by $\geq 80 \% .{ }^{4-10}$ Before ablation, variability of the PVC burden was assessed with 2 different Holter monitors $171 \pm 229$ days apart. No significant variability was found in the pre-ablation PVC burden $(\mathrm{P}=0.42)$. 
TABLE 1

Clinical Characteristics

\begin{tabular}{lc}
\hline Patients (\#) & 194 \\
Age & $49.9 \pm 14.3$ \\
Gender (M/F) & $91 / 103$ \\
PVC burden (\%) & $18.4 \pm 13.3$ \\
Symptomatic & $159 / 194(82 \%)$ \\
Ejection fraction (\%) & $56.4 \pm 8.4$ \\
Medications pre-ablation & \\
$\quad \beta$-Blocker & $121(62 \%)$ \\
Calcium channel blocker & $24(12 \%)$ \\
Antiarrhythmics & $29(15 \%)$ \\
Amiodarone & $7(4 \%)$ \\
Patients with previously failed procedures (n) & $42(22 \%)$ \\
\hline
\end{tabular}

*Anti-arrhythmics: propafenone, sotalol, mexiletine, flecainide.

\section{Electrophysiology Study}

After informed consent was obtained, conscious sedation and analgesia was administered with propofol, midazolam, fentanyl, and/or ketamine based on the attending physician's discretion and the patient';s wishes. For repeat procedures where a low intraprocedural PVC burden was felt to be the limiting factor, sedation was minimized and limited to propofol infusion with avoidance of midazolam or other longer acting sedatives. Multiple quadripolar electrode catheters were advanced via a femoral vein to the right ventricular apex, the His bundle position, and the high right atrium. After vascular access was obtained, 3,000 units of heparin were administered. If arterial access was necessary, additional heparin was administered to achieve an activated clotting time of 250-300 seconds. Surface electrograms were recorded in combination with bipolar intracardiac electrograms (EP Med Systems, West Berlin, NJ, USA).

In the absence of frequent PVCs, sedation was discontinued and isoproterenol was administered with a dose up to $20 \mathrm{mcg} / \mathrm{minute}$. Several maneuvers were performed to trigger PVCs: (1) atrial burst pacing, (2) ventricular burst pacing with cycle lengths of 500-200 milliseconds, (3) programmed ventricular stimulation with 4 extrastimuli ${ }^{11}$ at 3 drive cycle lengths of 350, 400, and 600 milliseconds. Atrial and ventricular pacing was performed during isoproterenol infusion and during isoproterenol withdrawal. Outcome at 3 months was analyzed depending on the PVC prevalence within the first 30 minutes of the procedure, independent of the success of provocative measures in triggering PVCs.

\section{Mapping and Ablation}

A 3-dimensional electroanatomical mapping system (CARTO, Biosense Webster, Diamond Bar, CA, USA) was used in all patients. Mapping was performed with a 3.5-mmopen-irrigation-tip catheter (Thermocool Navistar, Biosense Webster). Activation mapping was performed to identify the SOO of frequent PVCs. In the setting of infrequent ventricular ectopy, pace mapping was used to identify the SOO. At the SOO of a particular arrhythmia, pace mapping was performed and a matching pace map was identified when $\geq 10 / 12$ leads matched between the paced QRS complex and the targeted PVC.

An intramural focus was considered if mapping on either wall of the ventricular cavity showed early activation yet pace-mapping did not match with the targeted morphology of the PVC. An epicardial SOO was defined as a site within the coronary venous system or the epicardial space where the earliest activation time with a matching pace-map was identified.

Radiofrequency energy was delivered for 60-120 seconds at the SOO. An impedance drop of $10 \mathrm{ohms}$ was targeted with a power of 30-50 watts. If radiofrequency energy was delivered in the aortic cusps or within the coronary venous system, an initial power of 15-20 watts was used.

\section{Follow-Up}

Patients were seen in follow-up at 3-6 and 12-48 months postablation. Subsequently, patients were seen on an asneeded basis. Antiarhythmic medications were discontinued after an effective ablation procedure.

\section{Statistical Analysis}

Continuous variables are expressed as mean \pm 1 standard deviation and were compared with Student's $t$-test. Categorical variables were compared with the chi-square test. If the sample size was smaller than 5 in a given cell, Fisher's exact test was used. Logistic regression was used for multivariate analysis of independent variables. A P value of $<0.05$ was considered statistically significant. The PVC prevalence was quantified for the first 30 minutes of the procedure. A receiver operator characteristics (ROC) curve was created to assess whether there was a PVC burden that separated patients with successful ablation procedures from patients with a failed ablation procedure.

\section{Results}

\section{PVC Burden and Outcome}

A successful procedure was performed in 148 patients (76\%) resulting in a decrease in the PVC burden from 19.1 $\pm 13.6 \%$ to $0.38 \pm 0.98 \%(\mathrm{P}<0.0001)$ in these patients (Table 2). Initially, about one-third of patients (30\%) undergoing a PVC ablation procedure had infrequent intraprocedural PVCs and had a lower success rate than patients with frequent PVCs at the onset of the procedure (56\% vs. $85 \%$ respectively, $\mathrm{P}=0.0001$ ). A cut-off value of $32 \mathrm{PVCs} / 30 \mathrm{~min}$ utes best-separated patients with successful outcome from patients with a failed ablation (sensitivity: $78 \%$, specificity: $56 \%, \mathrm{P}<0.0001$, AUC $=0.68$, Fig. 1).

In patients with infrequent intraprocedural $\mathrm{PVCs}$, the PVC count during the initial 30 minutes was $7.9 \pm 10$ compared to $493 \pm 409$ in patients with frequent PVCs $(\mathrm{P}<0.0001)$

The mean PVC count during the first 30 minutes of the procedure was significantly higher in patients with a successful procedure than in patients who had a failed ablation procedure $(395 \pm 420$ vs. $194 \pm 327, \mathrm{P}=0.0001)$.

In patients with infrequent intraprocedural PVCs, the PVC burden was reduced from $11.4 \pm 10.4 \%$ to $3.9 \pm 7.6 \%(\mathrm{P}=$ $0.0001)$ at 3 months postablation. In patients with frequent intraprocedural PVCs, the PVC burden was reduced from $21.7 \pm 13.2 \%$ to $2.9 \pm 7.0 \%(\mathrm{P}=0.0001)$.

When the PVC burden during the first 30 minutes of the mapping procedure was compared to the preprocedure 24-hour Holter monitor, the burden was substantially lower in patients with infrequent intraprocedural PVCs $(0.38 \pm 0.49 \%$ intraprocedurally compared to $11.4 \pm 10.4 \%$ during the 
TABLE 2

Comparison of Patients with Frequent vs Infrequent PVCs

\begin{tabular}{|c|c|c|c|}
\hline Variable & Frequent PVCs & Infrequent PVCs & P Value \\
\hline Patients (\#) & $135(70 \%)$ & $59(30 \%)$ & \\
\hline Gender (M/F) & $65 / 70$ & $26 / 33$ & 0.6 \\
\hline Age (years) & $49.9 \pm 14.6$ & $49.7 \pm 13.7$ & 0.83 \\
\hline Symptoms (\%) & $108(80 \%)$ & $51(86 \%)$ & 0.28 \\
\hline Ejection fraction (\%) & $55.6 \pm 9.5$ & $58.3 \pm 4.5$ & 0.26 \\
\hline PVC-cardiomyopathy & $38(28 \%)$ & $7(12 \%)$ & 0.01 \\
\hline $\begin{array}{l}30 \text { minute } \\
\text { intraprocedural } \\
\text { PVC burden (\%) }\end{array}$ & $21.5 \pm 16.5$ & $0.38 \pm 0.49$ & 0.0001 \\
\hline $\begin{array}{l}\text { Holter PVC burden } \\
\text { pre-RF }(\%)\end{array}$ & $21.7 \pm 13.2$ & $11.4 \pm 10.4$ & 0.0001 \\
\hline \multicolumn{4}{|l|}{ Sedation/analgesia } \\
\hline Midazolam & $100(74 \%)$ & $37(63 \%)$ & 0.84 \\
\hline Propofol & $55(41 \%)$ & $22(37 \%)$ & 0.69 \\
\hline Fentanyl & $84(62 \%)$ & $27(46 \%)$ & 0.17 \\
\hline $\begin{array}{l}\text { Effective ablation at } 3 \\
\text { months }\end{array}$ & $115(85 \%)$ & $33(56 \%)$ & 0.0001 \\
\hline SOO & & & 0.67 \\
\hline Epicardial & $17(13 \%)$ & $8(14 \%)$ & \\
\hline Cusp & $17(13 \%)$ & $10(17 \%)$ & \\
\hline Papillary muscle & $15(11 \%)$ & $7(12 \%)$ & \\
\hline RVOT & $45(33 \%)$ & $27(46 \%)$ & \\
\hline Fascicular & $1(0.7 \%)$ & 0 & \\
\hline Intramural & $9(6.7 \%)$ & $1(1.7 \%)$ & \\
\hline LVOT & $12(8.8 \%)$ & $3(5.1 \%)$ & \\
\hline Parahisian & $6(4.4 \%)$ & $1(1.7 \%)$ & \\
\hline Mitral annulus & $6(4.4 \%)$ & $1(1.7 \%)$ & \\
\hline Tricuspid annulus & $3(2.2 \%)$ & 0 & \\
\hline LV posterobasal & $2(1.4 \%)$ & $1(1.7 \%)$ & \\
\hline LV posteroseptal & $1(0.7 \%)$ & 0 & \\
\hline $\begin{array}{l}\text { Aortomitral } \\
\text { continuity }\end{array}$ & $1(0.7 \%)$ & 0 & \\
\hline $\begin{array}{l}\text { Procedure time } \\
\text { (minutes) }\end{array}$ & $283.4 \pm 100.3$ & $289 \pm 98.5$ & 0.48 \\
\hline RF time (minutes) & $17.2 \pm 12.6$ & $17.1 \pm 15$ & 0.53 \\
\hline $\begin{array}{l}\text { Fluoroscopy time } \\
\text { (minutes) }\end{array}$ & $46.1 \pm 24$ & $45.1 \pm 23$ & 0.91 \\
\hline
\end{tabular}

$\mathrm{LV}=$ left ventricular; LVOT $=$ left ventricular outflow tract; $\mathrm{PVC}=$ premature ventricular complex; $\mathrm{RF}=$ radiofrequency ablation; $\mathrm{RVOT}=$ right ventricular outflow tract; $\mathrm{SOO}=$ site of origin.

24-hour Holter; $\mathrm{P}=0.0001)$. In patients with frequent intraprocedural PVCs, the PVC burden during the initial 30 minutes was no different compared to the preprocedural Holters $(21.5 \pm 16.5 \%$ intraprocedurally compared to $21.7 \pm 13.2 \%$ during the 24-hour Holter; $\mathrm{P}=0.70$ ).

In patients presenting with infrequent PVCs, there was no significant difference between the PVC burden pre-ablation when 2 preprocedural Holters were compared $(10.7 \pm 11.5 \%$ vs. $12.7 \pm 11.3 \%, \mathrm{P}=0.89)$. This was also the case for patients with frequent PVCs prior to the procedure (21.5 \pm $13.6 \%$ vs. $21.4 \pm 13.6 \%, \mathrm{P}=0.35$ ).

\section{Impact of Sedation and Analgesia}

Midazolam was used in 137 patients, propofol in 77 patients, fentanyl in 111 patients, and ketamine in 8 patients during the mapping procedure. There was no difference between infrequent and frequent intraprocedural PVC groups with respect to which, if any, sedatives, narcotics, or analgesics were given ( $\mathrm{P}$ values for midazolam, propofol, fentanyl, and ketamine were $0.84,0.69,0.17$, and 0.34 , respectively).

\section{Impact of Site of Origin}

The paucity of PVCs was associated with procedural failure independent of the SOO (OR: 6.9, 95\% CI: 3.0-16.2;
$P=0.0001)$. The sites of origin between intraprocedural infrequent and frequent PVC groups were similar $(\mathrm{P}=0.67)$. In 35 patients, PVCs originated from the epicardium or intramural sites. The success rates for these sites of origins was lower compared to other sites ( $57 \%$ vs. $81 \%$; $\mathrm{P}=0.003$ ). Furthermore, outcomes in this subset of patients were similar regardless of whether they had frequent or infrequent intraprocedural PVCs $(\mathrm{P}=0.37)$.

\section{Impact of Isoproterenol Administration}

In patients with infrequent PVCs, isoproterenol was administered. In patients in whom the PVC prevalence increased with isoproterenol (24/59), continued isoproterenol infusion was used to map the origins of PVCs. In these 24 patients, the success rate at 3 months was higher compared to patients in whom isoproterenol failed to increase the intraprocedural PVC prevalence $(79 \%$ vs. $42 \%, \mathrm{P}=0.008)$ and their outcome was similar compared to patients with frequent $\mathrm{PVCs}$ at the onset of the procedure ( $79 \%$ vs. $85 \%, \mathrm{P}=0.42)$.

\section{Yield of Pacing Maneuvers}

In 4/59 patients, pacing maneuvers resulted in a reproducibly inducible PVCs; however, these patients did not have a better outcome at 3 months compared to the other patients with frequent intraprocedural PVCs.

\section{Repeat Procedures}

Among the 19 patients with repeat procedures, 12 had infrequent PVCs during the index procedure and $3(15 \%)$ had infrequent PVCs during the repeat procedure. All 3 of these patients also had infrequent PVCs during the index procedure. A total 12 of 19 patients had effective ablation procedures and ablation failed in only one patient due to infrequent PVCs during the second procedure. In the remaining 6 patients with a failed procedure, the SOO could not be reached with the ablation catheter.

\section{Follow-Up}

Patients were followed for a mean of $19 \pm 18$ months after the ablation procedure. Nineteen patients required a repeat procedure. A second procedure was required more often in patients with infrequent intraprocedural PVCs compared to patients with frequent intraprocedural PVCs $(\mathrm{n}=12$ [20\%] vs. $\mathrm{n}=7$ [5.2\%], $\mathrm{P}=0.001$ ).

\section{Discussion}

\section{Main Findings}

This study demonstrates that the PVC burden within the first 30 minutes of the electrophysiology procedure impacted on the procedural outcome at 3 months. Patients with infrequent intraprocedural PVCs had a significantly lower success rate, independent of the SOO of PVCs, as compared to patients with frequent intraprocedural PVCs.

\section{PVC Burden During the Mapping Procedure}

In a subset of patients with frequent PVCs during baseline Holter, the PVC burden was substantially reduced at the onset of the mapping procedure. Day-to-day variability of the PVC burden might account for this observation; however, multiple preprocedural Holters failed to show a significant variability that might have accounted for the lack of PVCs in some 


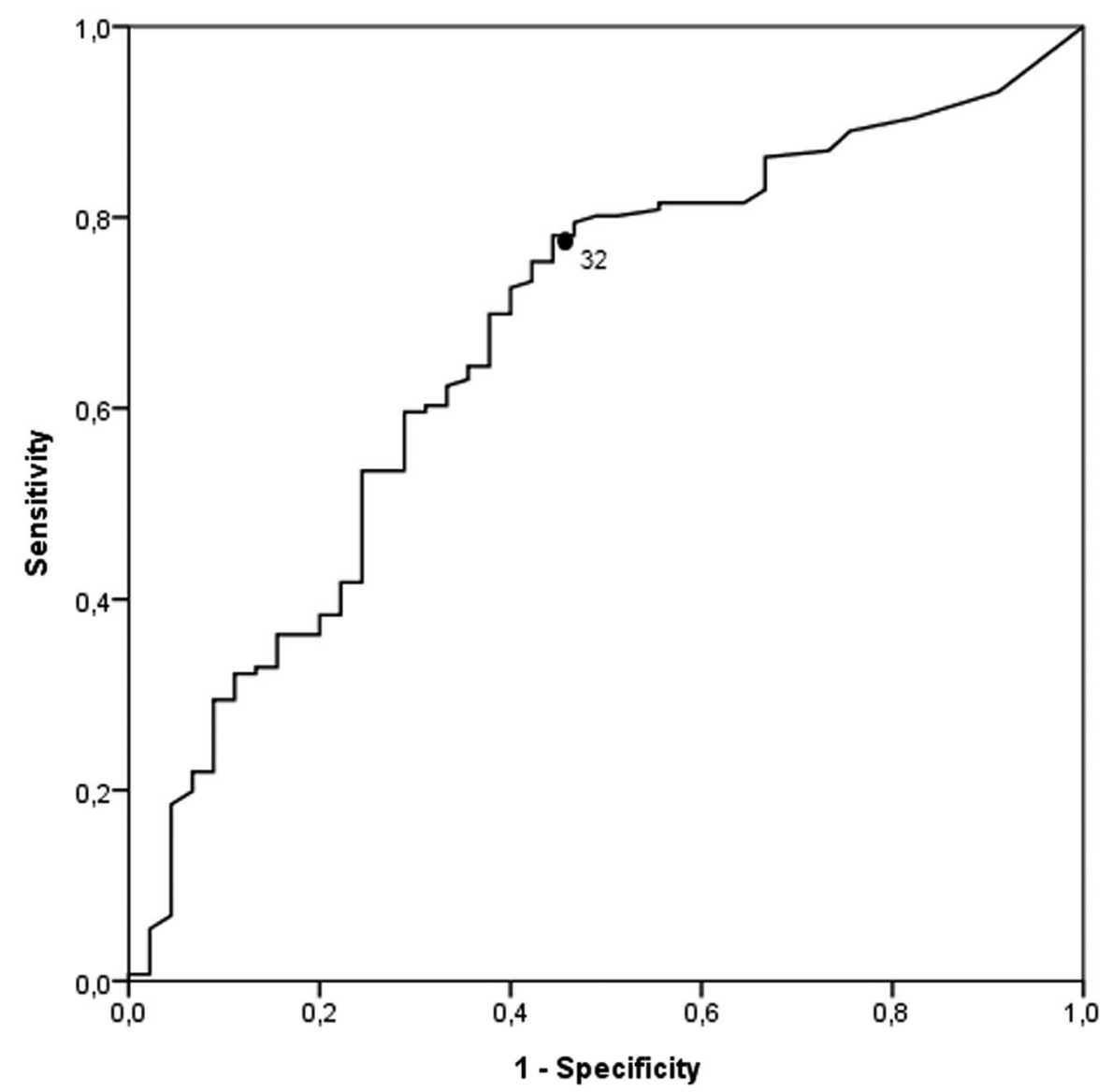

Figure 1. ROC analysis for the $P V C$ count within the first 30 minutes of the procedure. A PVC count $<32$ within the first 30 minutes of the procedure had a sensitivity of $78 \%$ and a specificity of $56 \%$ (area under curve: $0.68, P=0.0001$; 95\% CI: 0.59-0.78) differentiating patients with a successful procedure from patients with a failed procedure. patients at the onset of the procedure. Other factors might impact on the intraprocedural PVC prevalence. Hormones, particularly in women, may impact on PVC prevalence and may account for infrequent PVCs during parts of the menstrual cycle. ${ }^{12}$ However, there was no gender difference between patients with and without infrequent intraprocedural PVCs. Sedation can decrease spontaneous and inducible ventricular arrhythmias. ${ }^{13,14}$ However, sedation regimens were no different in patients with infrequent intraprocedural PVCs compared to patients with frequent intraprocedural PVCs. A higher susceptibility to sedation in some patients with frequent PVCs appears plausible and could have impacted on the procedural outcome. The baseline PVC burden in patients with infrequent intraprocedural PVCs was lower compared to patients in whom this was not the case and the lower burden might identify a population with a higher susceptibility to sedation. Longer acting sedatives like midazolam should be used with caution for sedation although in this study, patients exposed to midazolam were not more likely to have infrequent intraprocedural PVCs compared to patients in whom midazolam was not used. Nevertheless, when sedation was minimized during a second procedure in patients with unsuccessful ablations due to infrequent intraprocedural PVCs, PVCs were frequent enough to allow for identification of the SOO with activation mapping in most patients. The protocol of "minimal" sedation invoked among patients with repeat ablations included avoidance of longer acting sedatives like midazolam and use of short acting sedatives including propofol that could be titrated to the patients needs and could be discontinued in the absence of PVCs. This strategy resulted in a successful second ablation procedure in most patients.
With this strategy, the prevalence of patients with infrequent PVCs was cut in half during repeat procedures (from $30 \%$ to $15 \%$ ) despite that fact that most patients with repeat procedures had infrequent intraprocedural PVCs during the index ablation.

The lack of PVCs at the procedural onset could be overcome by administration of isoproterenol in some patients with infrequent PVCs and in these patients the outcome was similar compared to patients with frequent intraprocedural PVCs. Other maneuvers including pacing failed to improve outcome. This is important as the response to isoproterenol can be assessed prior to the electrophysiology procedure and predict likelihood of a successful ablation.

\section{Mapping Technique}

In the absence of frequent PVCs, pace-mapping is the predominant mapping technique. The spatial resolution and thereby the accuracy of pace-mapping has been demonstrated to be lower as compared to activation mapping. ${ }^{3}$ This study suggests that reliance primarily on pace-mapping results in a lower success rate most likely due to less precise mapping. Activation mapping is key in identifying the SOO in certain anatomic locations such as the aortic cusps where pacing with high output may be required in order to capture the ventricular myocardium. This may cause unintended capture of more distant myocardium resulting in an altered QRS morphology despite accurate catheter positioning at the SOO. For intramural sites of origin, activation mapping is equally important in order to identify endocardial breakthrough sites that are critical for selection of appropriate ablation sites. ${ }^{2}$ 


\section{Appropriate Ablation Endpoint}

The presence of frequent PVCs prior to radiofrequency ablation and the absence of PVCs postablation for a certain period of time is a reasonable endpoint of the ablation procedure. In the absence of frequent PVCs, ablation endpoints become less clear and this uncertainty may prompt the operator to deliver more ablation lesions.

\section{Limitations}

This is a retrospective analysis of a small series of patients and data will need to be collected prospectively to determine the exact impact of sedation on the intraprocedural PVC prevalence. However, patients with infrequent intraprocedural PVCs were given the same sedatives compared to patients with frequent intraprocedural PVCs arguing against the impact of sedation as the only factor for infrequent intraprocedural PVCs.

\section{Clinical Implications}

Frequency of intraprocedural PVCs impact on long-term success for ablation of idiopathic PVCs. If an ablation is performed in a patient with frequent PVCs who presents on the day of the procedure with infrequent PVCs, efforts should be maximized to increase the intraprocedural PVC burden. If this can be achieved with administration of isoproterenol, the success rate is similar to patients with frequent intraprocedural PVCs. Preprocedural monitoring and infusion of isoproterenol prior to the invasive procedure therefore may help to identify a group of patients in whom a mapping and ablation procedure will be beneficial. For repeat procedures, long acting sedatives should be avoided.

\section{References}

1. Baman TS, Ilg KJ, Gupta SK, Good E, Chugh A, Jongnarangsin K, Pelosi F Jr, Ebinger M, Crawford T, Oral H, Morady F, Bogun F: Mapping and ablation of epicardial idiopathic ventricular arrhythmias from within the coronary venous system. Circ Arrhythm Electrophysiol 2010;3:274-279.

2. Yokokawa M, Good E, Chugh A, Pelosi F Jr, Crawford T, Jongnarangsin K, Latchamsetty R, Oral H, Morady F, Bogun F: Intramural idiopathic ventricular arrhythmias originating in the intraventricular septum: Mapping and ablation. Circ Arrhythm Electrophysiol 2012;5:258-263.

3. Bogun F, Taj M, Ting M, Kim HM, Reich S, Good E, Jongnarangsin K, Chugh A, Pelosi F, Oral H, Morady F: Spatial resolution of pace mapping of idiopathic ventricular tachycardia/ectopy originating in the right ventricular outflow tract. Heart Rhythm 2008;5:339-344.

4. Morganroth J, Michelson EL, Horowitz LN, Josephson ME, Pearlman AS, Dunkman WB: Limitations of routine long-term electrocardiographic monitoring to assess ventricular ectopic frequency. Circulation 1978;58:408-414

5. Caron JF, Libersa CC, Kher AR, Kacet S, Wanszelbaum H, Dupuis BA, Poirier JM, Lekieffre JP: Comparative study of encainide and disopyramide in chronic ventricular arrhythmias: A double-blind placebo-controlled crossover study. J Am Coll Cardiol 1985;5:14571463.

6. Bogun F, Crawford T, Reich S, Koelling TM, Armstrong W, Good E, Jongnarangsin K, Marine JE, Chugh A, Pelosi F, Oral H, Morady F: Radiofrequency ablation of frequent, idiopathic premature ventricular complexes: Comparison with a control group without intervention. Heart Rhythm 2007;4:863-867.

7. Pool PE, Singh SN, Friedrich T: Effects of intravenous dofetilide in patients with frequent premature ventricular contractions: A clinical trial. Clin Cardiol 2000;23:415-416.

8. Baman TS, Lange DC, Ilg KJ, Gupta SK, Liu TY, Alguire C, Armstrong W, Good E, Chugh A, Jongnarangsin K, Pelosi F Jr, Crawford T, Ebinger M, Oral H, Morady F, Bogun F: Relationship between burden of premature ventricular complexes and left ventricular function. Heart Rhythm 2010;7:865-869.

9. Deyell MW, Park KM, Han Y, Frankel DS, Dixit S, Cooper JM, Hutchinson MD, Lin D, Garcia F, Bala R, Riley MP, Gerstenfeld E, Callans DJ, Marchlinski FE: Predictors of recovery of left ventricular dysfunction after ablation of frequent ventricular premature depolarizations. Heart Rhythm 2012;9:1465-1472.

10. Mountantonakis SE, Frankel DS, Gerstenfeld EP, Dixit S, Lin D, Hutchinson MD, Riley M, Bala R, Cooper J, Callans D, Garcia F, Zado ES, Marchlinski FE: Reversal of outflow tract ventricular premature depolarization-induced cardiomyopathy with ablation: Effect of residual arrhythmia burden and preexisting cardiomyopathy on outcome. Heart Rhythm 2011;8:1608-1614.

11. Hummel D, Strickberger S, Daoud E, Niebauer M, Bakr O, Man K, Williamson B, Morady F: Results and efficiency of programmed ventricular stimulation with four extrastimuli compared with one, two, and three extrastimuli. Circulation 1994;90:2827-2823.

12. Marchlinski FE, Deely MP, Zado ES: Sex-specific triggers for right ventricular outflow tract tachycardia. Am Heart J 2000;139:10091013.

13. Joshi S, Wilber DJ: Ablation of idiopathic right ventricular outflow tract tachycardia: Current perspectives. J Cardiovasc Electrophysiol 2005;16(Suppl 1):S52-S58.

14. Aliot EM, Stevenson WG, Almendral-Garrote JM, Bogun F, Calkins CH, Delacretaz E, Della Bella P, Hindricks G, Jais P, Josephson ME, Kautzner J, Kay GN, Kuck KH, Lerman BB, Marchlinski F, Reddy V, Schalij MJ, Schilling R, Soejima K, Wilber D: EHRA/HRS expert consensus on catheter ablation of ventricular arrhythmias: Developed in a partnership with the European Heart Rhythm Association (EHRA), a registered branch of the European Society of Cardiology (ESC), and the Heart Rhythm Society (HRS); in collaboration with the American College of Cardiology (ACC) and the American Heart Association (AHA). Heart Rhythm 2009;6:886-933. 